\title{
Groundwater flow below construction pits and erosion of temporary horizontal layers of silicate grouting
}

\author{
Joris M. Dekker ${ }^{1} \cdot$ Thomas Sweijen $^{1,2} \cdot$ Alraune Zech $^{1}$ \\ Received: 24 April 2020 / Accepted: 17 September 2020 / Published online: 1 October 2020 \\ (C) The Author(s) 2020
}

\begin{abstract}
Injection of silicate grouting materials is widely used to create temporary horizontal layers for reducing inflow of groundwater at construction sites, in regions with shallow water tables. The erosion of a grouting layer was investigated by means of analytical solutions for groundwater flow and transport within a pit after construction finished. Erosion is assumed to occur by dissolution of the temporary injection layer and subsequent advective transport. Thereby, the hydraulic conductivity changes with time. This paper presents novel analytical solutions and approximate solutions for the major fluxes in the construction pit as a function of the domain settings, aquifer gradient and hydraulic conductivity. In addition, the mass flux and the dilution ratio of erosion-related components leaving the construction pit and entering the aquifer are quantified. Derived solutions are verified against numerical simulations. A sensitivity study shows the impact of domain settings on fluxes and dilution ratio. The results confirm that mass flux of grout components increases with ongoing erosion. Thus, its effect on groundwater quality increases with time after construction ceased.
\end{abstract}

Keywords Silicate grouting $\cdot$ Erosion $\cdot$ Flow barriers $\cdot$ Analytical solutions $\cdot$ Contamination

\section{Introduction}

Over the last decades, urbanization resulted in an increasing demand in space for infrastructure within already densely populated areas. Therefore, many infrastructures are developed within the subsurface such as drainage systems, underground public transport networks, roads and parking facilities. Realizing these structures often require working below the water table. This requires sealing and dewatering of excavation sites, whilst keeping the impact on the surrounding aquifer to a minimum.

A well-established method to build an excavation site in urban areas of shallow-groundwater is by combining vertical

Electronic supplementary material The online version of this article (https://doi.org/10.1007/s10040-020-02246-3) contains supplementary material, which is available to authorized users.

Thomas Sweijen

T.Sweijen@uu.nl

1 Department of Earth Sciences, Environmental Hydrogeology Group, Utrecht University, Princetonlaan 8a, 3584CB Utrecht, The Netherlands

2 Crux Engineering BV, Amsterdam, The Netherlands soil-retaining walls and lowering the water table by pumping within these walls (Azzam 2004, Powers et al. 2007; Pujades et al. 2014). However, large-scale dewatering activities lower the water table beyond the excavation site, having negative environmental impact - for example, pumping induced drawdowns (in the order of meters) cause settlement of the soil (e.g. Spacagna et al. 2017) or displacement of contaminants which are often present in urban aquifers (Fetter et al. 2018). Moreover, shallow aquifers are nowadays used for subsurface geothermal systems (e.g. ATES) which can be affected by dewatering activities causing a loss in thermal efficiency (Bloemendal and Hartog 2018).

Alternative methods to dewatering are the usage of soilinjections of silicate grouting material and application of underwater concrete. The latter creates a permanent horizontal layer between the vertical walls. Whereas, application of silicate grouting materials reduces the permeability/hydraulic conductivity of the soil layer temporarily (Littlejohn et al. 1997; Van der Stoel 2001). The appropriate method to use varies for each project, but remains a balance between costs, environmental impact and feasibility.

Silicate groutings are popular because they are costefficient and avoid large-scale dewatering. For instance, in the 1990 s more than $100,000 \mathrm{~m}^{3}$ of silicate grouting was applied in Berlin, Germany (Eiswirth et al. 1997). A typical 
grouting injection is a mixture of sodium silicate solution, water and a solidifier. During injection, the silica remains in solution due to a high $\mathrm{pH}$ value caused by caustic soda (Littlejohn et al. 1997). After injection, sodium silicate starts forming a pore-filling gel as result of the solidifier (Owusu 1982; Littlejohn et al. 1997). The gel eventually clogs the pore-space between the sand grains. Consequently, soil stabilizes and the hydraulic conductivity is reduced for several orders of magnitude (Kazemian et al. 2010). During construction, water is pumped without impacting the aquifer due to the hydraulically decoupling, whereas after construction is finished, dewatering stops and the remaining pit is refilled with water. As a result of groundwater flow, the grout material in the temporary injection layer (abbreviated hereafter as "til") is slowly dissolved. Dissolved components of this erosion process are transported with the groundwater into the aquifer.

Although injections of sodium silicate solutions are widely used, little is known about the dynamics and the environmental impact of its erosion products on the surrounding aquifer. They pose a risk to the groundwater quality and potentially to surrounding subsurface energy systems. Sodium silicate injections are associated with an increase in $\mathrm{pH}$ and a change in groundwater composition (Schnell 2001), which can lead to precipitation of iron oxides at extraction wells and subsequent well clogging (De Zwart 2007). An increase in $\mathrm{pH}$ can also lead to enhanced concentrations of dissolved organic matter (Curtin et al. 2016; Schnell 2001) being an additional thread to extraction wells. Eiswirth et al. (1997); Eiswirth and Hötzl (2003), and Schnell (1999) studied the environmental impact of tils in a German aquifer. They report an elevation in dissolved organic matter, $\mathrm{pH}$, sodium and silica occurring up to $10 \mathrm{~m}$ downstream. Given that in these studies vertical sheetpiling was removed after construction, the til eroded swiftly (in about 4 years). However, tils are often realized between permanent vertical walls, being part of the building foundation. This significant different flow configuration around the til leads to a slower erosion mechanism (more in the range of a decade).

Comprehensive studies are missing which quantify the erosion based on the physical processes involved: transient groundwater flow; advective, diffusive and dispersive transport; chemical reactions; and adsorption/desorption. A challenge here is the changing hydraulic conductivity of the til due to dissolution and thus permanent change in the underlying hydraulic head and flow pattern. Numerical models may be used to quantify transport of dissolution products at specific sites; however, in practice conducting a comprehensive study is usually not feasible due to limited resources. In this line, simple analytical expressions are highly valuable to overcome the limitation of characterizing flow and transport as function of known domain and aquifer parameters. They can be used in practice to estimate groundwater flow and mass fluxes without much effort.
Here, flow and erosion of a til between permanent vertical walls is quantified using a simplified approach. A set of analytical solutions is derived for hydraulic heads and groundwater fluxes in a two-dimensional (2D) domain with a til of temporally variable hydraulic conductivity. In addition, a dilution ratio is specified which enables to quantify the environmental impact of erosion products on groundwater leaving the domain. Approximate analytical solutions are verified against numerical solution. Without doing a detailed site-specific (numerical) research, these novel solutions provide estimates for fluxes and concentration for any field setting, being highly valuable for practical purposes.

\section{Flow model and settings}

\section{Conceptual model}

This paper investigates groundwater flow inside the remainder of a construction pit of a subsurface building structure in a shallow sandy aquifer as displayed schematically in Fig. 1. The site is assumed to be fully saturated with groundwater since dewatering stopped after construction finished.

The construction pit represents a typical setting in the Netherlands. The subsurface construction is surrounded by vertical walls such as Cutter soil mix (CSM) walls that are part of the building foundation. The lifetime of these walls is supposed to be more than 100 s of years. The walls are therefore considered permanent (see e.g. Lin et al. 2016). In between the vertical walls, the til is realized. It is placed at a considerable depth below the final building floor to ensure hydraulic stability during construction; the groundwater water pressure from below the til needs to be compensated by the overburden of the soil on top of the til. After construction is finished, the til remains in place, but is slowly eroded. The characteristic time-scale of grout erosion relates to the residence time of groundwater within the construction pit which can be estimated as ratio of the construction pit length and groundwater flow velocity. For typical settings in the Netherlands, this leads to a time frame of a few years up to a decade.

The domain is conceptualized as a two-dimensional (2D) cross-section (Fig. 1) with a width $L$ and a height $T$. The two vertical walls are located at $x=0$ and $x=L$ and act as no-flow boundaries (Fig. 2). The til is located at height $H$, has a thickness $D$, and a hydraulic conductivity of $K_{\text {til }}$, which varies over time. The aquifer has a constant hydraulic conductivity $K_{\text {sand }}$ and is assumed to be homogeneous. The same holds for the soil above the til. The pit consists of three horizons: (1) the area between the til and the bottom of the vertical walls; (2) the area of the til (in place or eroded); (3) the area above the til (Fig. 1). The segmentation refers to the areas of distinct hydraulic conductivity with the first and the last relating to $K_{\text {sand }}$ 


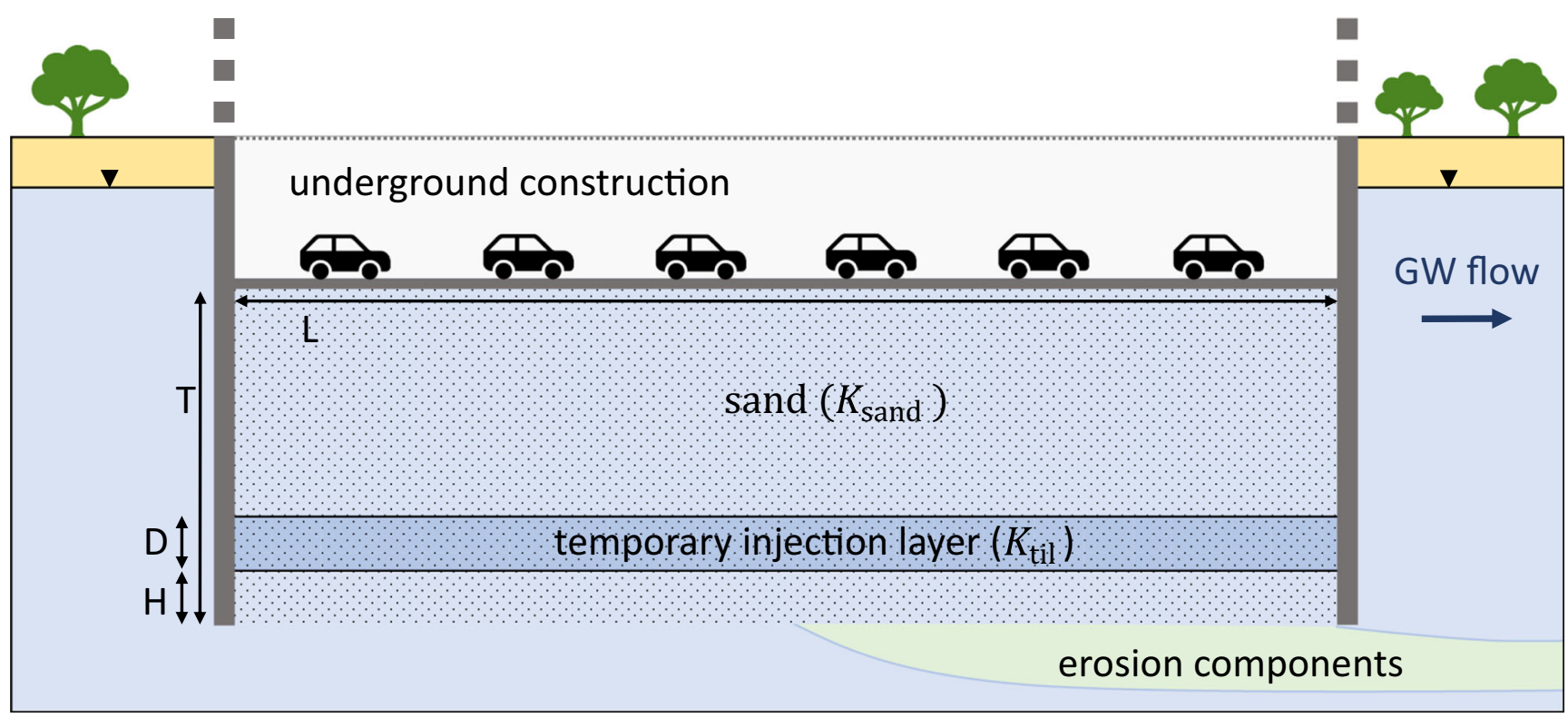

Fig. 1 Conceptual model of construction pit (dotted area) within an aquifer. Setting parameters are specified in Table 1. Thick gray lines mark impermeable vertical concrete walls. Groundwater $(\mathrm{GW})$ flow is

and the second to $K_{\text {til }}$. The groundwater flow below the construction pit is assumed constant following a linear head gra$\operatorname{dient} A$. A set of default parameters is defined in Table 1 . They represent realistic values for construction pits and head gradients in the Netherlands. The hydraulic conductivity of the sand is based on data from the Dutch Geological Survey (DINOloket 2019). Note that default values are used for comparison of numerical and analytical solutions, but the results are not limited to the values listed in Table 1 .

\section{Erosion states and time}

Investigation starts when the construction is finished: dewatering stops and the remaining setting is refilled with groundwater. The til is still in place and has a very low from left to right following a linear head gradient. Erosion components leave at the right half of the domain following the flow pattern in the pit (Fig. 2)

hydraulic conductivity $K_{\text {til }}^{0}$. Erosion of the til starts due to dissolution of silicate grouting into groundwater. This timedependent process has two bounds: the initial state $(t=0)$ and the final state $(t=\infty)$. During erosion, the hydraulic conductivity of the til increases until it is the same as that of sand: $K_{\text {til }}^{\infty}=K_{\text {sand. }}$. The final state $(t=\infty)$ can have grout material present, but it has no effect on the hydraulic conductivity such that the construction pit is homogeneous again. Intermediate times relate to conductivity values in between $K_{\text {til }}^{0}<K_{\text {til }}<K_{\text {sand }}$.

Firstly, this paper mathematically investigates the two specified states of the til at $t=0$ and $t=\infty$, by deriving for each case analytical solutions for hydraulic head distributions and fluxes (section 'Analytical fluxes for limiting states'). Secondly, this paper presents a newly developed approximate

Table 1 Default values of construction site parameters used for the numerical model

\begin{tabular}{lll}
\hline Parameter & Variable symbol & Default value \\
\hline Aquifer hydraulic conductivity & $K_{\text {sand }}$ & $25 \mathrm{~m} / \mathrm{day}$ \\
Initial hydraulic conductivity in til & $K_{\text {til }}^{0}$ & $8.6 \times 10^{-3} \mathrm{~m} / \mathrm{day} \quad\left(=1 \times 10^{-7} \mathrm{~m} / \mathrm{s}\right)$ \\
Length of the excavation pit & $L$ & $50 \mathrm{~m}$ \\
Total depth & $T$ & $10 \mathrm{~m}$ \\
Depth of til & $H$ & $1 \mathrm{~m}$ \\
Thickness of til & $D$ & $1 \mathrm{~m}$ \\
Hydraulic gradient in the aquifer & $A$ & $-3.8 \times 10^{-4} \mathrm{~m} / \mathrm{m}$ \\
Absolute hydraulic head & $B$ & $0.287 \mathrm{~m}$ \\
Groundwater velocity (porosity of 0.35$)$ & $v$ & $\sim 10 \mathrm{~m} /$ year \\
Residence time of groundwater $(\mathrm{gw})$ below pit & $t_{\mathrm{gw}}$ & 5 years \\
\hline
\end{tabular}

For local specification see Figs. 1 and 2 (til temporary injection layer) 

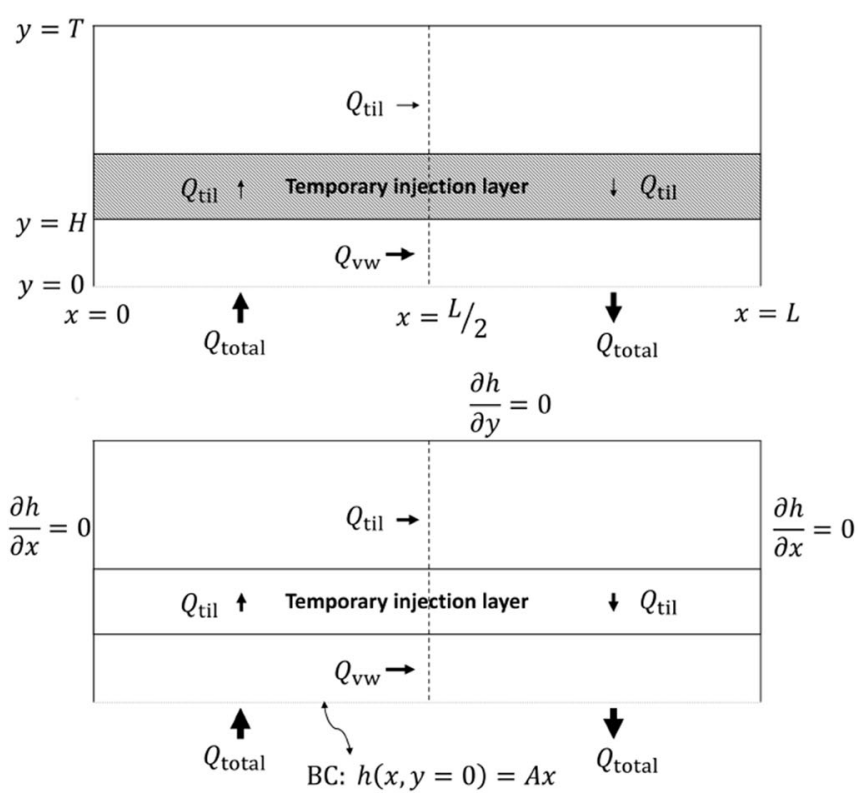

Fig. 2 a-d Flow situations at the beginning $t=0$ (top row, $\mathbf{a}-\mathbf{b}$ ) and the end $t=\infty$ (bottom row, $\mathbf{c}-\mathbf{d}$ ) of til erosion. The left side (a, c) shows the scheme of fluxes within the construction pit: Total flux in and out of the domain $Q_{\text {total }}$, flux below the til $Q_{v w}$ and flux through and above the til $Q_{\text {til. }}$. The thickness of the arrows indicates strength of flux. The domain

analytical expression for intermediate times (section 'Flux approximation for intermediate times').

In this work, transient groundwater flow is not considered, as would be formally necessary for taking the temporally increasing conductivity $K_{\text {til }}$ of the grout layer into account. Instead, a series of steady-state situations is investigated for various conductivity distributions in the pit. This simplification is based on the assumption that heads immediately adapt to erosion induced changes in the hydraulic conductivity. This is warranted given that dynamic effects in head distributions have a reference time on the order of days, while erosion of the til has a reference time on the order of years, being proportional to the residence time of groundwater (Table 1).

\section{General mathematical model}

For every state of the til, i.e. every value of $K_{\text {til }}$, the conceptual model refers to a steady-state groundwater-flow situation in a layered medium. It is described by Laplace's equation:

$\frac{\partial}{\partial x}\left(K(x, y) \frac{\partial h(x, y)}{\partial x}\right)+\frac{\partial}{\partial y}\left(K(x, y) \frac{\partial h(x, y)}{\partial y}\right)=0$

Here $h(x, y)$ is the hydraulic head distribution and $K(x, y)$ is the spatially heterogeneous hydraulic conductivity in the construction pit, representing the layered structure (Fig. 2a). Equation (1) can be solved exactly using a numerical model (section 'Modflow model and error quantification'); however, this work applies a simplifying assumption for $K(x, y)$ to
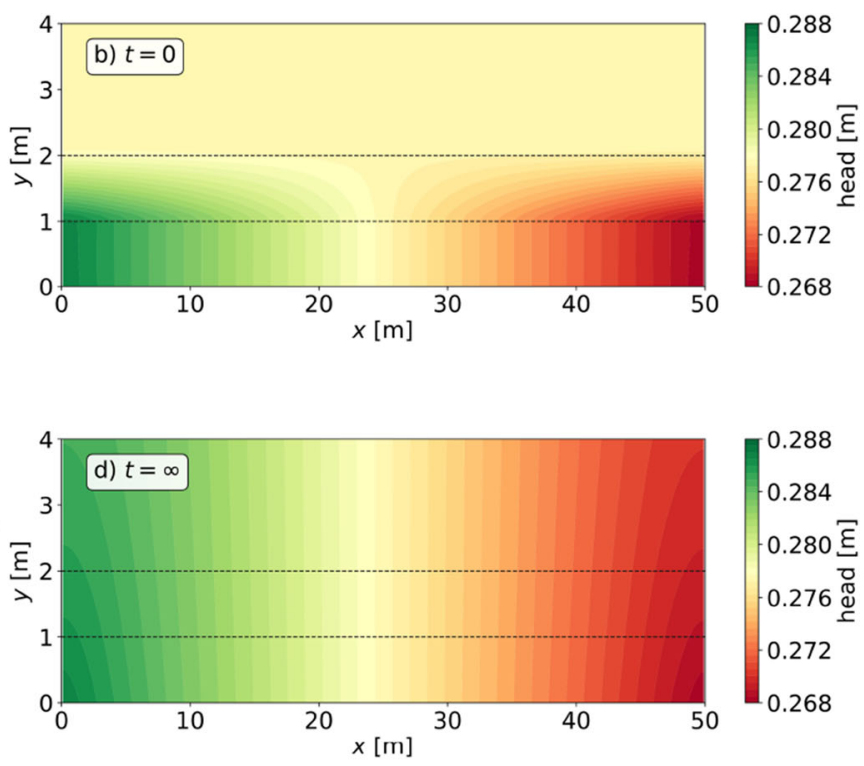

coordinates (a) and boundary conditions (c) are outlined and labelled. On the right side $(\mathbf{b}, \mathbf{d})$ are corresponding contour plots of hydraulic head distribution (from the numerical model) for the standard parameters (Table 1). Dashed lines indicate location of the til. Plots only show the lowest $4 \mathrm{~m}$ of the construction site for better visualization

enable derivation of analytical expressions (section 'Analytical solutions for limiting states').

No flow boundary conditions are present along the walls and at the top of the construction site: $\frac{\partial}{\partial x} h(L, y)=0$ and $\frac{\partial}{\partial x} h$ $(0, y)=0$ for $0<y<T$ as well as $\frac{\partial}{\partial y} h(x, T)=0$ for $0<x<L$. A constant head gradient is located at the contact plane to the aquifer: $h(x, 0)=A x+B$ for $0<x<L$ (Fig. 2c).

Hydraulic head distributions $h(x, y)$ as solutions of Eq. (1) given the specified boundary conditions are visualized in Fig. $2 \mathrm{~b}, \mathrm{~d}$ for the two limiting cases of hydraulic conductivity in the til, initial state $(t=0)$ and the final state $(t=\infty)$ of erosion.

\section{Defining fluxes}

The left column of Fig. 2 shows a schematic overview of the groundwater fluxes within the construction pit. Fluxes are spatially distributed given the non-uniform head distribution. The focus is on three major fluxes within the domain:

- $Q_{\text {total }}\left[\mathrm{m}^{2} /\right.$ day]: the total flux that enters the construction pit from below, which is an integrative measure of the inflowing water over the left domain half $(0<x<L / 2$ at $y=0)$. Given the water balance it is the same amount of outflowing water over $L / 2<x<L$.

- $Q_{\text {til }}\left[\mathrm{m}^{2} /\right.$ day]: the flux through the til; which integrates the flow along $0<x<L / 2$ at $y=H$. Given the mass balance, $Q_{\mathrm{til}}$ is also the total flux above the injection layer over $H<$ 
$y<T$ at $x=\mathrm{L} / 2$ and back through the til over $L / 2<x<L$ at $y=H$.

- $Q_{\mathrm{vw}}\left[\mathrm{m}^{2} /\right.$ day]: the flux between the vertical walls below the til, which integrates the flow along $0<y<H$ at $x=\mathrm{L} / 2$.

The different fluxes are particularly important to quantify dilution of dissolved til-components. The amount of grout material dissolved out of the til is related to $Q_{\text {til. }}$. The flux $Q_{\mathrm{vw}}$ determines how much this amount is diluted with fresh groundwater. $Q_{\text {total }}$ provides information about the amount of total flux leaving the construction pit.

Given the groundwater volume balance, the fluxes relate as: $Q_{\mathrm{total}}=Q_{\mathrm{til}}+Q_{\mathrm{vw}}$. Each of the three fluxes is constant for a fixed conductivity distribution within the pit. However, the strength of each flux depends on $K_{\text {til }}$, thus the state of erosion of the til. Note, that a distinction is made in the flux for all erosion states, even for the final state. Although conductivity has increased to that of the aquifer then, dissolved material of the til can still be present in the groundwater flow leaving the construction pit.

\section{MODFLOW model and error quantification}

A numerical model was set up to calculate exact values for the head and fluxes within the construction pit for various settings of the parameters, particularly the hydraulic conductivity of the til. To this end, MODFLOW 2005 (Harbaugh 2005) is employed by using Python package Flopy (Bakker et al. 2016). MODFLOW solves the groundwater flow equation (Eq. 1) in the layered domain with the specified boundary conditions using a finite difference method. A uniform grid size is applied of $0.125 \mathrm{~m}$. Default values for domain size and hydraulic conductivities are listed in Table 1. The use of Flopy allows to easily adjust simulation to different parameter values.

Results of the numerical model are considered as exact solutions for head and fluxes. They are used to determine the relative error $\epsilon=\left|\left(Q^{\text {num }}-Q^{\text {ana }}\right) / Q^{\text {num }}\right|$ between numerically determined quantities $\left(Q^{\text {num }}\right)$ and analytical expressions $\left(Q^{\text {ana }}\right)$. This metric serves as test for the validity of approximation. A deviation of less than $10 \%$ is considered to be acceptable and a deviation below is considered $5 \%$ as good.

\section{Fluxes and transport}

\section{Analytical fluxes for limiting states}

The groundwater flow Eq. (1), in combination with the boundary conditions, can be solved analytically for particular cases of hydraulic conductivity distributions $K(x, y)$. This work presents analytical solutions for $h(x, y)$ and subsequently for fluxes $Q$ for two limiting states of the til, at $t=0$ (layered domain) and $t=\infty$ (homogeneous domain). Detailed derivations are provided in the electronic supplementary material (ESM). They follow standard mathematical procedures for solving partial differential equations.

\section{The homogeneous case}

At the final stage of erosion $(t=\infty)$, the conductivity in the til has reached that of the sand $\left(\lim _{t \rightarrow \infty} K_{\text {til }}=K_{\text {sand }}\right)$, thus the domain has returned to a homogeneous state. An analytical solution for the hydraulic head of Laplace's equation for this setting is:

$h(x, y)=A L\left(0.5-\frac{4}{\pi^{2}} \sum_{i=1}^{\infty} \frac{\cos ((2 i-1) \pi \widetilde{x}) \cdot \cosh ((2 i-1) \pi \widetilde{T}(\widetilde{y}-1))}{(2 i-1)^{2} \cosh ((2 i-1) \pi \widetilde{T})}\right)+B$

where $\widetilde{x}=\frac{x}{L} \in[0,1]$ and $\tilde{y}=\frac{y}{T} \in[0,1]$ are the dimensionless coordinates in horizontal and vertical direction, scaled by length $L$ and height $T$ of the construction pit; $\widetilde{T}=T / L$ can be interpreted as the domain ratio. $A$ is the hydraulic gradient below the pit and $B$ is the absolute head at the lower left domain $(x=0, y=0)$.

Equation (2) is a fully exact solution for the considered case. As further proof, the analytical solution (Eq. 2) is compared to the numerical solution of the MODFLOW model. As expected, results differ less than $1 \%$. Figure 3 provides a visual proof.

Fluxes for this setting can be determined from Darcy's Law and integration over the appropriate domain part (section 'General mathematical model'). The total flux $Q_{\text {total }}^{\infty}$ results in a similar infinite sum as Eq. (2), which can be simplified to:

$Q_{\text {total }}^{\infty}=-c_{1} \frac{4 K_{\text {sand }} A L}{\pi^{2}} \tanh \left(c_{1} \pi \widetilde{T}\right)$

The coefficient here is $c_{1}=0.90$. The simple expression in Eq. (3) differs less than 1\% from the exact solution. A detailed discussion on the solution can be found in the ESM.

The flux through the til can be similarly determined making use of the stream functions as:

$Q_{\mathrm{til}}^{\infty}=-c_{1} \frac{4 K_{\mathrm{sand}} A L}{\pi^{2}}\left(\tanh \left(c_{1} \pi \widetilde{T}\right) \cdot \cosh \left(c_{1} \pi \widetilde{H}\right)-\sinh \left(c_{1} \pi \widetilde{H}\right)\right)$

where $\widetilde{H}=H / L$ is the dimensionless height of til and the optimal coefficient is $c_{1}=0.90$. Again, the expression in Eq. (4) differs less than $1 \%$ from the exact solution being expressed as an infinite sum (again see ESM for more details).

The horizontal flux below the til follows from the water balance as $Q_{\mathrm{vw}}=Q_{\mathrm{total}}-Q_{\mathrm{til}}$. A simplified expression is:

$Q_{\mathrm{vw}}^{\infty}=-c_{1} \frac{4 K_{\mathrm{sand}} A L}{\pi^{2}} \tanh \left(c_{1} \pi \widetilde{H}\right)$ 

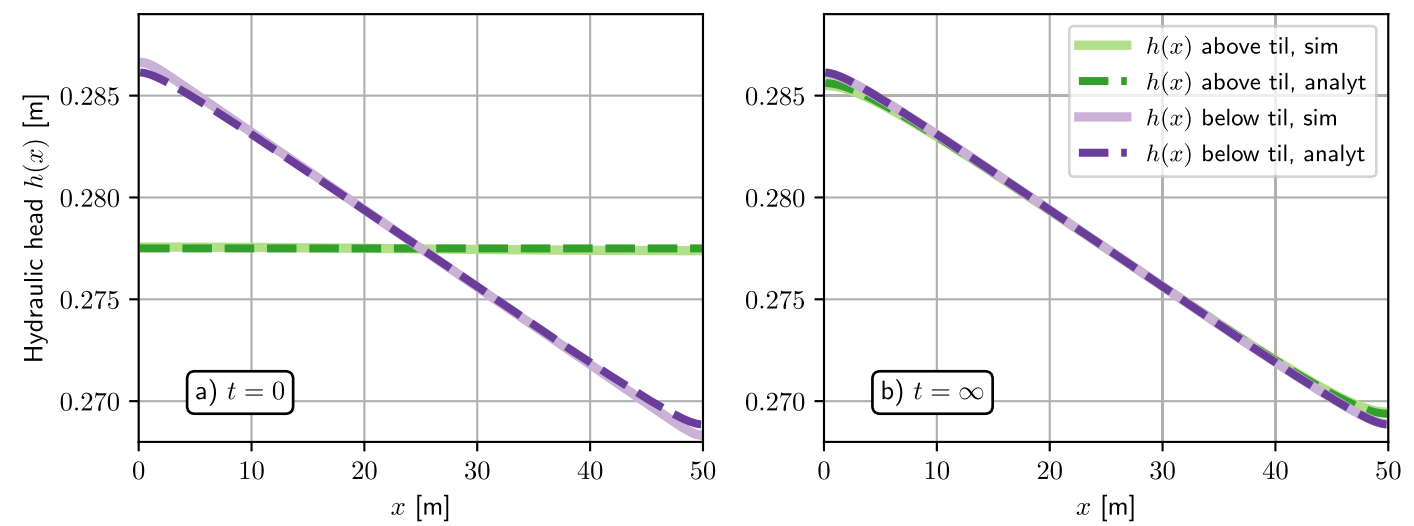

Fig. 3 Hydraulic head profiles $h(x)$ along the length of the construction pit: located just below (purple line, $y=D$ ) and just above (green line, $y=D+H$ ) the til at the a beginning $(t=0)$ and $\mathbf{b}$ end $(t=\infty)$ of erosion. Numerical results in solid lines, analytical results in dashed lines

All fluxes are directly proportional to the hydraulic head gradient $A$ and the hydraulic conductivity $K_{\text {sand }}$ of the aquifer. Thus, the flux increases with higher gradients and higher conductivities linearly. Given their exactness, the analytical expressions (Eqs. 3-5) are valid for any choice of parameters beyond the values listed in Table 1 .

\section{The layered case}

Before erosion starts, at $t=0$, the hydraulic conductivity within the domain is layered, due to the lower value of the til (inhomogeneous domain). Instead of solving Eq. (1) for the layered hydraulic conductivity distribution, approximate solutions are presented for head and fluxes, where details on derivations are presented in the ESM. The following observations from the numerical model are made (Fig. 3): (1) the horizontal head gradient above the til is approximately zero due to the hydraulic decoupling by the low conductivity of the til; (2) the head profile directly below the til at $y=H$ is similar to the head profile below the construction pit.

The domain is conceptually divided into a lower part, the til and an upper part. Segments are treated independently. The flux below the til $Q_{\mathrm{vw}}$, refers to the total flux within the lower part, being a homogeneous domain with depth $H$. Using the results of the previous section (Eq. 3), the following expression is obtained:

$Q_{\mathrm{vw}}^{0}=-c_{1} \frac{4 K_{\mathrm{sand}} A L}{\pi^{2}} \tanh \left(c_{1} \pi \widetilde{H}\right)$

The flux $Q_{\text {til }}^{0}$ into the upper segment is approximated by assuming that flow through the til is only vertical. The head gradient above the til is zero and the gradient directly below the til equals that at the inlet of the pit. Making use of Darcy's Law and integration (see ESM) provides:

$Q_{\mathrm{til}}^{0}=-\frac{K_{\mathrm{til}}^{0} A L^{2}}{8 D}$
Here $K_{\text {til }}^{0}$ denotes the initial hydraulic conductivity in the til and $\mathrm{D}$ is the til layer thickness. The total flux into the domain then results with $Q_{\mathrm{total}}^{0}=Q_{\mathrm{vw}}^{0}+Q_{\mathrm{til}}^{0}$. For very small ratios $\frac{K_{\mathrm{til}}^{0}}{K_{\mathrm{sand}}}$ of conductivity, $Q_{\mathrm{total}}^{0}$ basically equals $Q_{\mathrm{vw}}^{0}$, since the flux throughout the til is negligible given its impermeability.

Comparison of the head profiles obtained with the approximate analytical head and those from the MODFLOW model in Fig. 3 show almost perfect agreement indicating the validity of the simplifications. The relative error between approximate analytical expressions (Eqs. 6 and 7) and numerically determined fluxes are below $3 \%$ for all fluxes using the default parameters. The critical aspect here is the ratio of the hydraulic conductivity of the til and the hydraulic conductivity of sand. The approximations are valid as long as the ratio $\frac{K_{\mathrm{til}}^{0}}{K_{\mathrm{sand}}}$ is small.

A parameter sensitivity study is performed to identify the range of applicability of the approximate analytical fluxes, using dimensions of typical construction pits beyond their default values (Table 1). For each parameter combination, a relative error is determined of all fluxes, for variable length of the construction pit $L$, various heights $D$ and thicknesses $D$ of the til. Details, including a graphical representation of the parameter sensitivity study can be found in the ESM. The relative errors for all fluxes are acceptable (even below $3 \%$ ) for most parameter values. Highest sensitivity showed the flux through the til $Q_{\mathrm{til}}^{0}$. It starts to deviate by more than $5 \%$ for very narrow $(L<15 \mathrm{~m})$ or extremely long construction pits $(L>100 \mathrm{~m})$; deeply placed vertical walls compared to the til $(H>3.5 m)$ or very thin til $(D<0.35 \mathrm{~m})$. However, all settings with deviations above $5 \%$ are rarely encountered in practice.

In general, the analytical expressions for the fluxes $Q_{\mathrm{vw}}^{0}$ and $Q_{\text {til }}^{0}$ represent the flow situation in typical construction pits shortly after construction finished. However, the solutions for the fluxes are not valid at later times where erosion of the til lead to higher conductivities and a less prominent layering of the domain. The fluxes for these intermediate 
stages of erosion are presented in section 'Flux approximation for intermediate times'.

\section{Mass flux and dilution ratio}

The til is a source for solutes that are being transported out of the construction pit into the aquifer. Actual erosion of the til is a complex, transient and spatially distributed process depending on the chemical settings and structure of the silicate grouting as well as on the prevailing groundwater flow conditions. In the following, only advective transport is considered, thus the focus is on transport of solutes out of the til and construction pit as well as major flow and transport patterns. Transport by diffusion and dispersion is neglected, which is justified by their much smaller scale.

This paper considers homogeneous erosion, where concentrations of dissolved components of the til are spatially and temporally constant. In contrast, heterogeneous erosion would account for variability in effluent concentration caused, e.g. by preferential flow due to weaknesses in the layer structure or temporal changes in dissolution behavior. Homogeneous erosion is exemplified by the work of Schnell (2001), where erosion of a silicate grouting was studied in groundwater in field and laboratory, in a flow tank. Schnell (2001) reports an initial increase of concentration with a brief peak, associated to flushing out of silicate grouting material that did not solidify. Then, in the erosion phase, concentrations became constant in time with values in the range of $200-300 \mathrm{mg} / \mathrm{L}$. The observation of Schnell (2001) are adapted in this work by considering a constant concentration $C_{\max }$ of erosion products in the til. $C_{\max }$ is related to the maximal concentration determined by solubility, residence time of groundwater within the til and the various chemical interactions of groundwater with the til.

For homogeneous erosion, the mass flux of grout material out of the til can be considered as product of the groundwater flux through the til $\mathrm{Q}_{\text {til }}$ and the constant concentration in the til $C_{\text {max }}$ :

$F=C_{\text {max }} Q_{\text {til }}$

Essential to the environmental impact of erosion is the concentration that leaves the construction pit $C_{\text {out }}$. Erosion-related products undergo dilution before entering the aquifer. The mass flux out of the pit is $F=C_{\text {out }} Q_{\text {total }}$. Given mass conservation, the total mass flux $F$ is constant and the concentration of erosion products out of the pit follows with:

$C_{\text {out }}=\frac{Q_{\text {til }}}{Q_{\text {total }}} C_{\text {max }}=\mu C_{\text {max }}$

Here, the dilution ratio $\mu$ is sometimes referred to as a dilution factor, in its form initial to total amount:
$\mu=\frac{Q_{\mathrm{til}}}{Q_{\mathrm{total}}}=1-\frac{Q_{\mathrm{vw}}}{Q_{\mathrm{total}}}$

Through the definition of the fluxes, $\mu$ is a sole function of the construction pit settings: total depth $T$, length $L$ and depth of the til $D$. The dilution ratio $\mu$ describes not only dilution of eroded components in the groundwater, but it also determines the chemical interaction between erosion products (alkalinity, sodium, dissolved silica, etc.) with groundwater. The dilution ratio allows for calculations on the buffering capacity of the groundwater to accommodate the erosion products.

\section{Flux approximation for intermediate times}

Of particular interest is the erosion behavior at intermediate times, i.e. in between the limiting cases of $t=\infty$ (homogeneous) and $t=0$ (layered domain with noneroded til). To derive an approximate description of fluxes, the solutions for the both limiting cases are interpolated. Interpolation is based on the hydraulic conductivity of the til $K_{\text {til }}$, which increases with time. Therefore, the hydraulic conductivity ra-

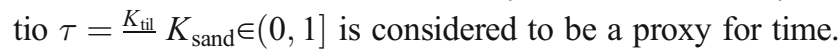
Finding a direct relation between $\tau$ and $t$ requires transient reactive transport simulations with gradually increasing hydraulic conductivities, which is beyond the scope of this work.

This work studies the time-dependent erosion through steady state MODFLOW simulations with gradually increasing value of $K_{\text {til }}$ starting at the almost impermeable value $K_{\text {til }}$ $=K_{\text {til }}^{0}(t=0)$ and ending with that of the aquifer $K_{\text {til }}=K_{\text {sand }}$ $(t=\infty)$. At each value of $K_{\mathrm{til}}$, the steady-state head and flow fields are numerically computed from which the fluxes $Q_{\text {til }}$, $Q_{\mathrm{vw}}$ and $Q_{\text {total }}$ are determined. Figure 4 shows the simulated fluxes as function of the ratio of conductivity in the til and the constant aquifer conductivity $K_{\text {sand }}$.

The flux below the til $\left(Q_{\mathrm{vw}}\right)$ is quasi constant with increasing $K_{\text {til }}$. Thus, the flux below the til is considered to be independent of time and $Q_{\mathrm{vw}}(\tau) \approx Q_{\mathrm{vw}}^{0}$ as given in Eq. (6).

The total flux $Q_{\text {total }}$ scales with increasing conductivity ratios in a logarithmic relation, which can be seen in the logscale display of Fig. 4. Based on these findings, an interpolating function is defined for the total flux as linear function in log-scale:

$Q_{\text {total }}(\tau)=\left(Q_{\mathrm{vw}}+Q_{\text {til }}^{0}-Q_{\text {total }}^{\infty}\right) \cdot \frac{\log (\tau)}{\log \left(\tau_{0}\right)}+Q_{\text {total }}^{\infty}$

Here $Q_{\mathrm{vw}}$ (Eq. 6) and $Q_{\text {til }}^{0}$ (Eq. 7) are the fluxes below and through the til, respectively. They sum up to the total flux $Q_{\text {total }}^{0}=Q_{\mathrm{vw}}+Q_{\mathrm{til}}^{0}$ into the construction pit at initial state. $Q_{\text {total }}^{\infty}$ is the total flux into the homogeneous domain (Eq. 3) and $\tau_{0}=\frac{K_{\text {til }}^{0}}{K_{\text {sand }}}$ is the conductivity ratio at initial state. The function in Eq. (11) is displayed in Fig. 4. 

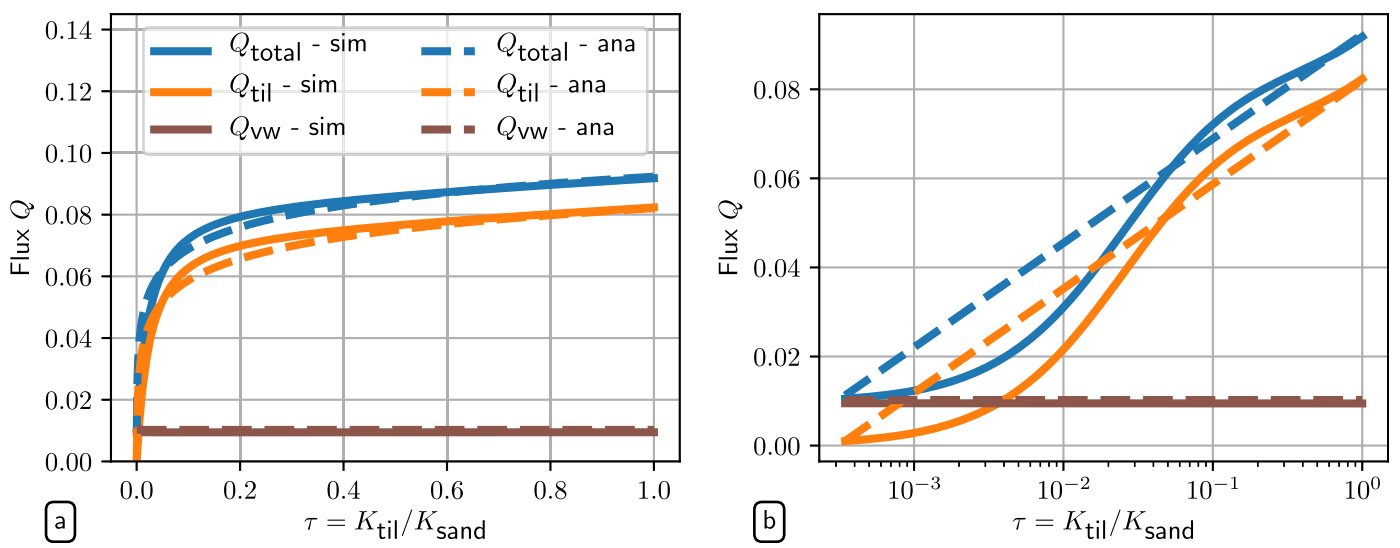

Fig. 4 Fluxes through the domain as function of hydraulic conductivities ratio $\tau$ as proxy for the temporal evolution of the grout erosion process in a linear and $\mathbf{b}$ log-scale. Solid lines show numerically determined fluxes, dashed lines represent approximate transition solution as defined in Eq. (11)

The flux $Q_{\text {til }}(\tau)$ is the difference between the total flux $Q_{\text {total }}(\tau)$ and the flux below the til: $Q_{\mathrm{til}}(\tau)=Q_{\mathrm{total}}(\tau)-Q_{\mathrm{vw}}$. Since $Q_{\mathrm{vw}}$ is constant, $Q_{\mathrm{til}}(\tau)$ has the same logarithmic scaling as $Q_{\text {total }}$ and can be similarly approximated making use of a log-linear function as displayed in Fig. 4.

The evaluation of the relative difference between the fluxes $Q(\tau)$ determined numerically and through the empirical expressions showed deviations in the range of 5\% or less for all fluxes and intermediate times proving it a reasonable approximation.

The dilution ratio as a function of pseudo time $\tau$ follows with

$$
\begin{aligned}
\mu(\tau)= & 1-\frac{Q_{\mathrm{vw}}}{Q_{\mathrm{total}}(\tau)} \\
= & \frac{\left(Q_{\mathrm{vw}}+Q_{\mathrm{til}}^{0}-Q_{\mathrm{total}}^{\infty}\right) \cdot \log \left(\frac{\tau}{\tau_{0}}\right)+Q_{\mathrm{til}}^{0} \cdot \log \left(\tau_{0}\right)}{\left(Q_{\mathrm{vw}}+Q_{\mathrm{til}}^{0}-Q_{\mathrm{total}}^{\infty}\right) \cdot \log (\tau)+Q_{\mathrm{total}}^{\infty} \cdot \log \left(\tau_{0}\right)}
\end{aligned}
$$

Figure 5 shows the analytical approximation of the dilution ratio (Eq. 12) compared to exact results from numerical simulations. The analytical expression underestimates the dilution ratio for very small ratios $\tau$, i.e. values of $K_{\text {til }}$ close to the initial conductivity $K_{\text {til }}^{0}$. For ratios up to $\tau=1: 100$, the analytical

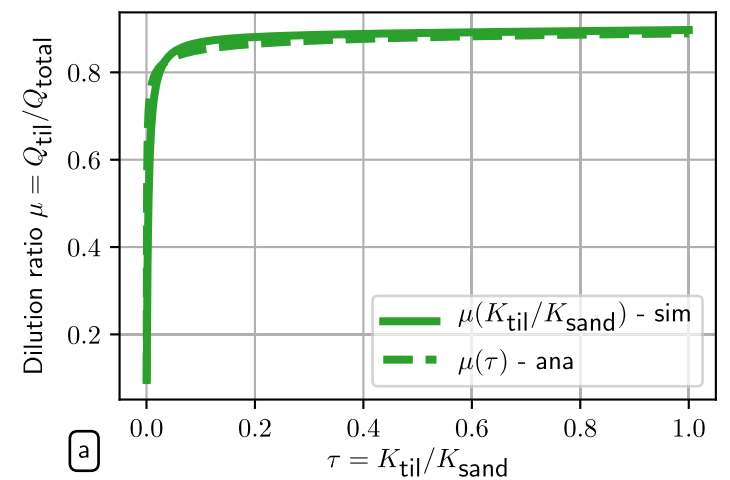

solution overestimates the dilution ratio. However, the linear display of $\mu(\tau)$ reveals that the general trend is well captured. Relative differences between numerical solutions and the approximate analytical expression are within the acceptable range of $10 \%$.

\section{Results}

\section{Dependency of mass fluxes on hydraulic conductivity ratio}

Increasing the hydraulic conductivity $k_{\text {til }}$ causes the flux through the til $\left(Q_{\text {til }}\right)$ and the flux out of the construction pit $Q_{\text {total }}$ to increase (Fig. 4). In contrast, the flux below the til remains quasi constant. As a result, $Q_{\text {til }}$ becomes larger than $Q_{\mathrm{vw}}$ in early stages of erosion; already at small ratios of $\tau=$ $K_{\mathrm{til}} / K_{\text {sand }}$. The contribution of $Q_{\mathrm{vw}}$ to the total flux becomes less significant the longer erosion continues, which is also confirmed by the numerical solution (solid lines, Fig. 4). The empirical solution Eq. (11) reproduces this general behavior as log-linear function (dashed lines, Fig. 4).

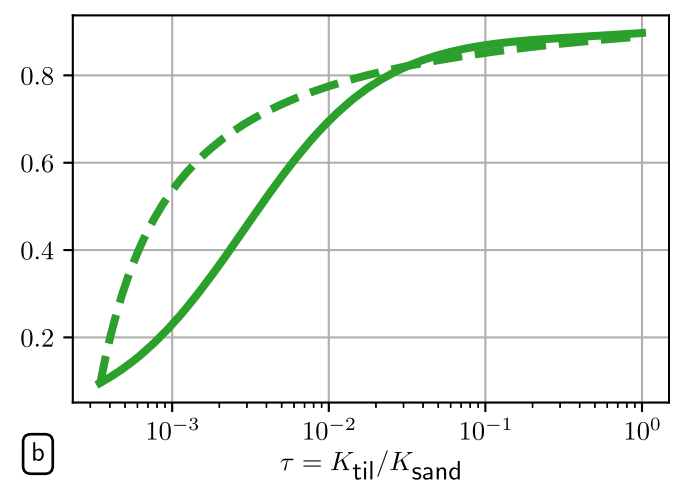

Fig. 5 Dilution ratio $\mu(\tau)$ as function of the hydraulic conductivity ratio of the til and that of the domain $\tau=K_{\text {til }} / K_{\text {sand }}$ in a linear and $\mathbf{b}$ log-scale. Solid lines show numerically determined fluxes, dashed lines represent approximate transition solution as defined in Eq. (12) 
The effect of increasing $\tau=K_{\text {til }} / K_{\text {sand }}$ is also apparent in the dilution ratio $\mu(\tau)$ as displayed in Fig. 5: $\mu$ increases with time due to the increase in $Q_{\text {til }}$. The dilution of the flux out of the til quickly reduces with increasing erosion of the til. The numerical simulations show that the dilution ratio approaches 1 for ratios of $\tau=\frac{K_{\text {til }}}{K_{\text {sand }}}>\frac{1}{100}$, indicating little dilution of the flux out of the construction pit. A typical threshold value of 1:10 for the dilution ratio is exceeded already for low values of $K_{\text {til }}$. Thus, significant amounts of erosion products leave the construction pit with possibly harmful effects to the aquifer.

Potentially negative effects of erosion to the groundwater quality increase with time. The mass flux into the aquifer is highest not directly after construction ceased, but a long time after. However, this work only considers homogeneous erosion with constant concentration. With ongoing erosion, concentrations of solutes are likely to drop as the residence time of water in the til decreases and grout material is depleted. At that point, mass flux will decrease again, which is not covered by the approach in this paper.

\section{Dependency of mass fluxes on domain settings}

The maximum possible mass flux $F$ out of the construction pit is directly proportional to the flux $Q_{\text {total }}=F / C_{\text {out }}$, which is a function of the domain settings. Figure 6 shows an analysis of the fluxes as function of the ratios of total depth $T$ to length $L$
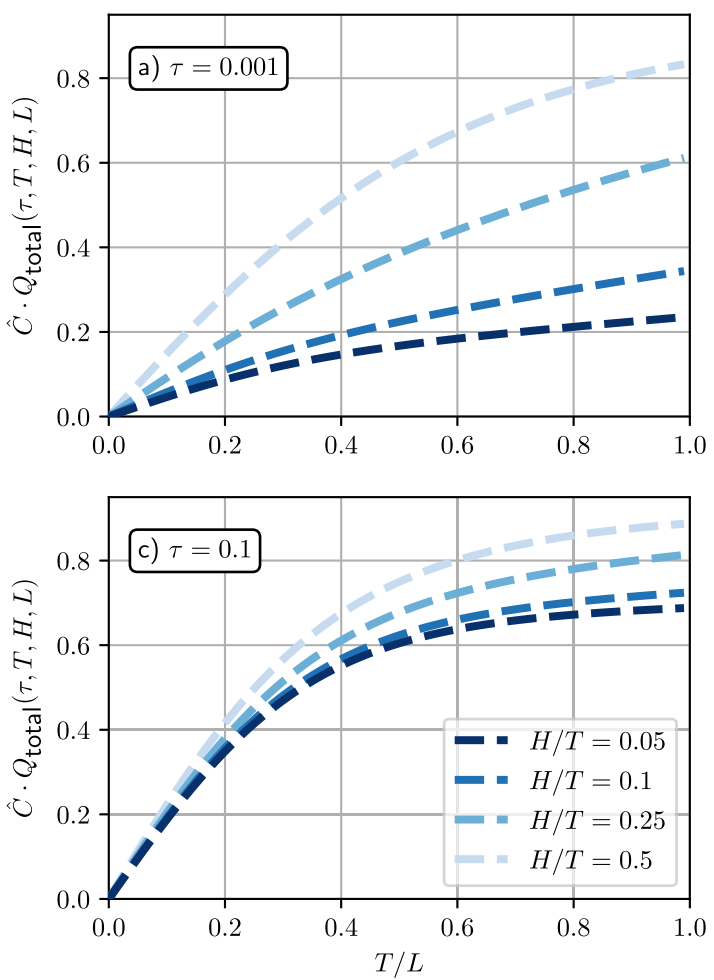

Fig. 6 a-d Dependency of $Q_{\text {total }}$ (Eq. 11) on setting parameters of the construction pit for increasing hydraulic conductivity ratios $\tau=K_{\mathrm{til}} / K_{\mathrm{sand}}$ : $T / L$ is the ratio of total depth $T$ to total length $L$ of the domain; $H / T$ denotes and of $T$ to depth of the til $H$ for several ratios $\tau=\frac{K_{\text {til }}}{K_{\text {sand }}}$ of hydraulic conductivity, equivalent to different times in the erosion process. Note that displayed $Q_{\text {total }}$ is basically independent of the default parameters defined in Table 1.

$Q_{\text {total }}$ increases asymptotically with increasing depth to length ratio $T / L$. For deep til (small $H / T$ ), the value of flux increased with ongoing erosion (increasing $\tau$ ). For shallow til, the total flux is just slightly impacted by the increase of conductivity in the til, since the flux through it is generally small. Here, although the total flux is higher, the amount of effluent out of the pit is lower given a higher dilution.

The flux through the til, $Q_{\text {til }}$ shows a similar asymptotical behavior as $Q_{\text {total }}$ with increasing ratio $T / L$ (Fig. 7). The flux decreases for large depth to length ratios, especially for high $\tau$ values. In contrast to $Q_{\text {total }}$, differences given different depth ratios $H / T$ amplify with ongoing erosion. Particularly for deep tils (small $H / T$ ), $Q_{\text {til }}$ is almost as high as $Q_{\text {total }}$, giving little dilution of the mass flux.

$Q_{\text {til }}(\tau)$ is also a function of the til thickness $D$. By testing its impact, $Q_{\text {til }}$ is found to decrease with increasing $D$, thus allowing less flux through the til due to a stronger sealing effect. This is the results of the sensitivity of $Q_{\mathrm{til}}^{0}$ toward $D$, as discussed in section 'The layered case' (and the ESM).

Figure 8, showing the dilution ratio for the same settings as in Figs. 6 and 7, confirms that dilution increase with increasing depth of the til (smaller $H / T$ ). This effect is independent of
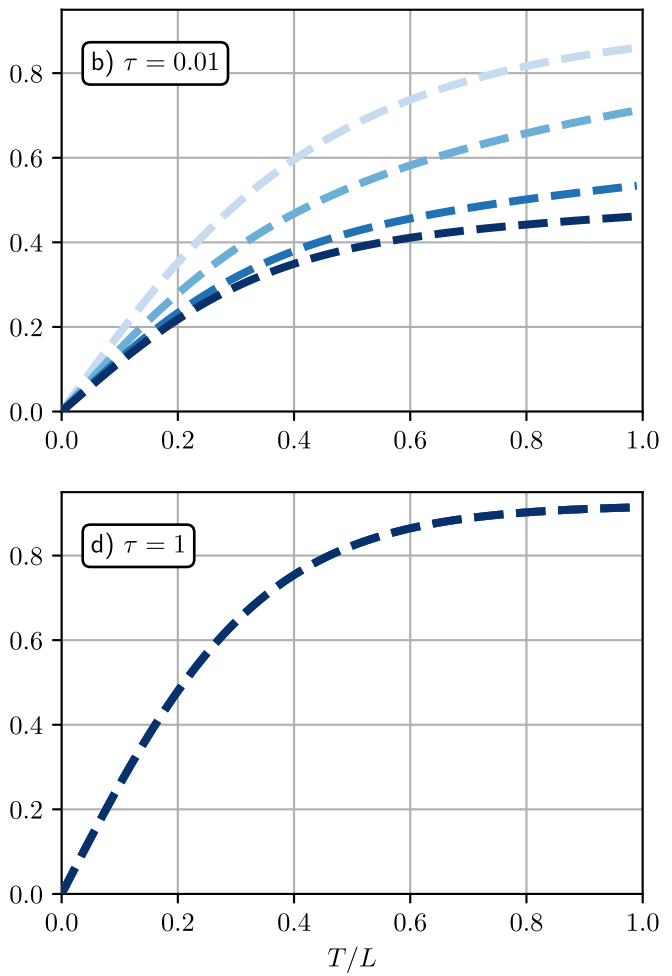

the depth of the til $H$ to total depth $T$. The prefactor $\widehat{C}=\pi^{2} /\left(4 A L K_{\text {sand }}\right)$ makes the expression dimensionless 

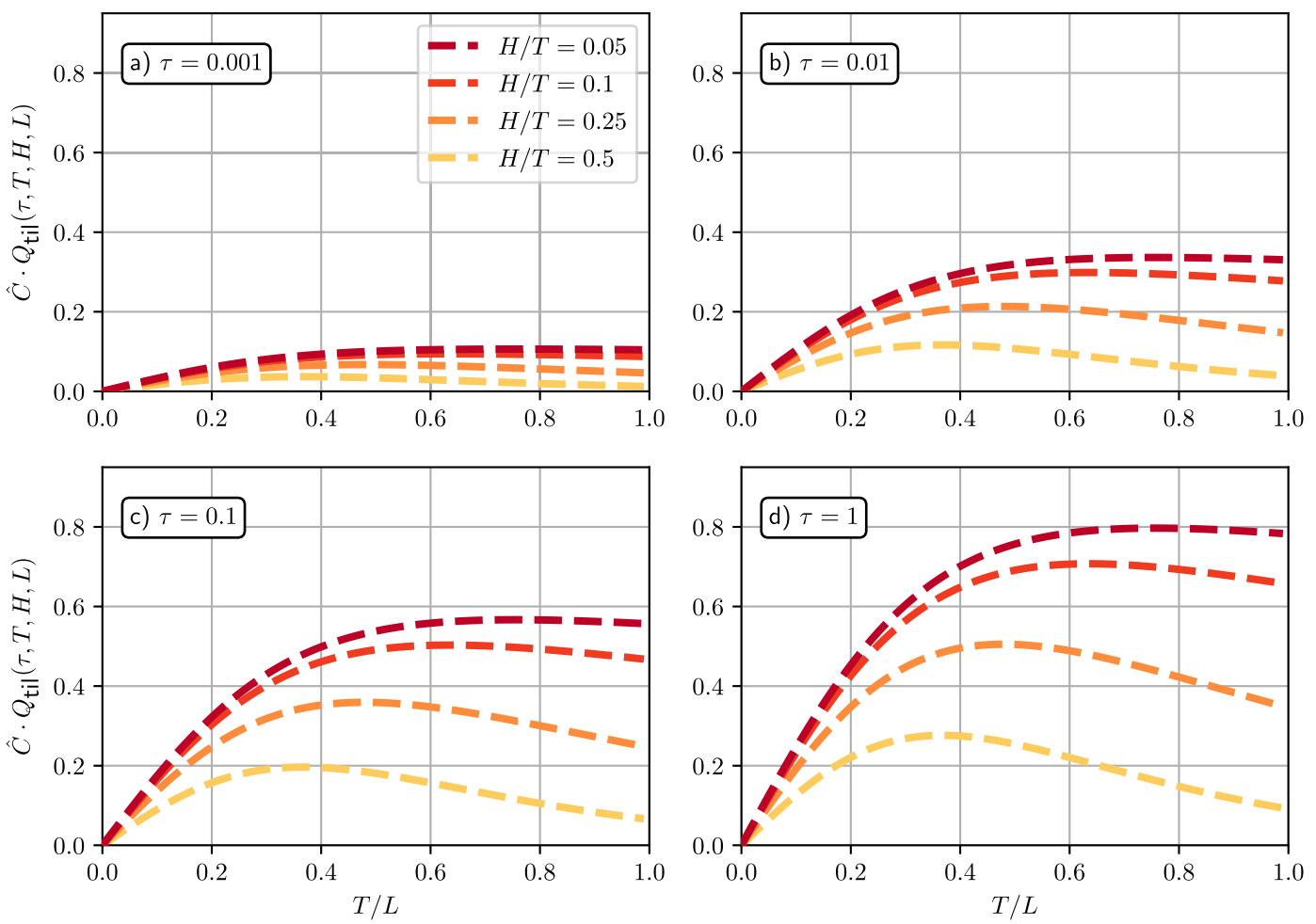

Fig. 7 a-d Dependency of $Q_{t i l}$ on setting parameters of the construction pit for increasing hydraulic conductivity ratios $\tau=K_{\text {til }} / K_{\text {sand }}: T / L$ is the ratio of total depth $T$ to total length $L$ of the domain; $H / T$ denotes the depth

of the til $H$ to total depth $T$. The prefactor $\widehat{C}=\pi^{2} /\left(4 A L K_{\text {sand }}\right)$ makes the expression dimesionless

the total depth to length ratio $T / L$ as well as of the ratio of conductivities $\tau$, although amplifying with ongoing erosion.

In summary, a reduction of groundwater flow through the til and consequently a reduction in mass flux into aquifer is obtained by: (1) decreasing the total depth of the domain $T$, i.e. shorter vertical walls for a larger depth to length ratio $T / L ;(2)$ increasing the depth of the til $H$, which increases dilution within the construction pit; (3) a thicker til, i.e. increasing $D$. However, the latter is also related to the injection of more grout material resulting in higher costs and a prolonged erosion process.

\section{Summary and conclusions}

This paper studies the characteristics of saturated groundwater flow within a construction pit to assess the erosion of silicate grouting after the construction. Analytical expressions are derived for the hydraulic head distribution and groundwater fluxes within a construction pit, which contains a horizontal temporary injection layer (til) of distinct hydraulic conductivity. Heads and fluxes are expressed as function of the 2D domain parameters (depth, length), the location of the til (depth, thickness) and aquifer parameters such as hydraulic gradient and conductivity. Solutions are presented for the initial and final state of the system, when the til is still intact (very low hydraulic conductivity), and when the til is eroded (same hydraulic conductivity as the

surrounding soil), respectively. Since the hydraulic conductivity of the til increases with ongoing erosion, this ratio is considered as a proxy for time. In this line, a log-linear interpolation function is presented for fluxes and dilution ratio as approximate analytical expression for the temporal erosion behavior. Mass flux of eroded silicate grouting from the construction site into the aquifer is the product of groundwater fluxes and dissolved concentrations, considering homogeneous erosion. The maximum mass flux is reached when the hydraulic conductivity in the til approaches that of the aquifer. Analytical solution further allowed to performed a sensitivity study determining how fluxes and dilution ratio change as function of the domain dimensions. Numerical simulations providing exact fluxes confirm that the approximate analytical solutions have a relative error of less than $5 \%$.

The results in this paper lead to the following conclusions:

- Total inflow of groundwater into the construction pit is dominated by the contrast of hydraulic conductivity between aquifer and the til of silicate grouting. The flux below the silicate grout layer is quasi constant. The total groundwater flux into and out of the pit increases with the amount of flux through the injection layer. Both fluxes increase exponentially when the conductivity of the layer approaches that of the aquifer.

- The same holds for the dilution ratio. The mass flux of grout components is diluted initially, but dilution reduces 

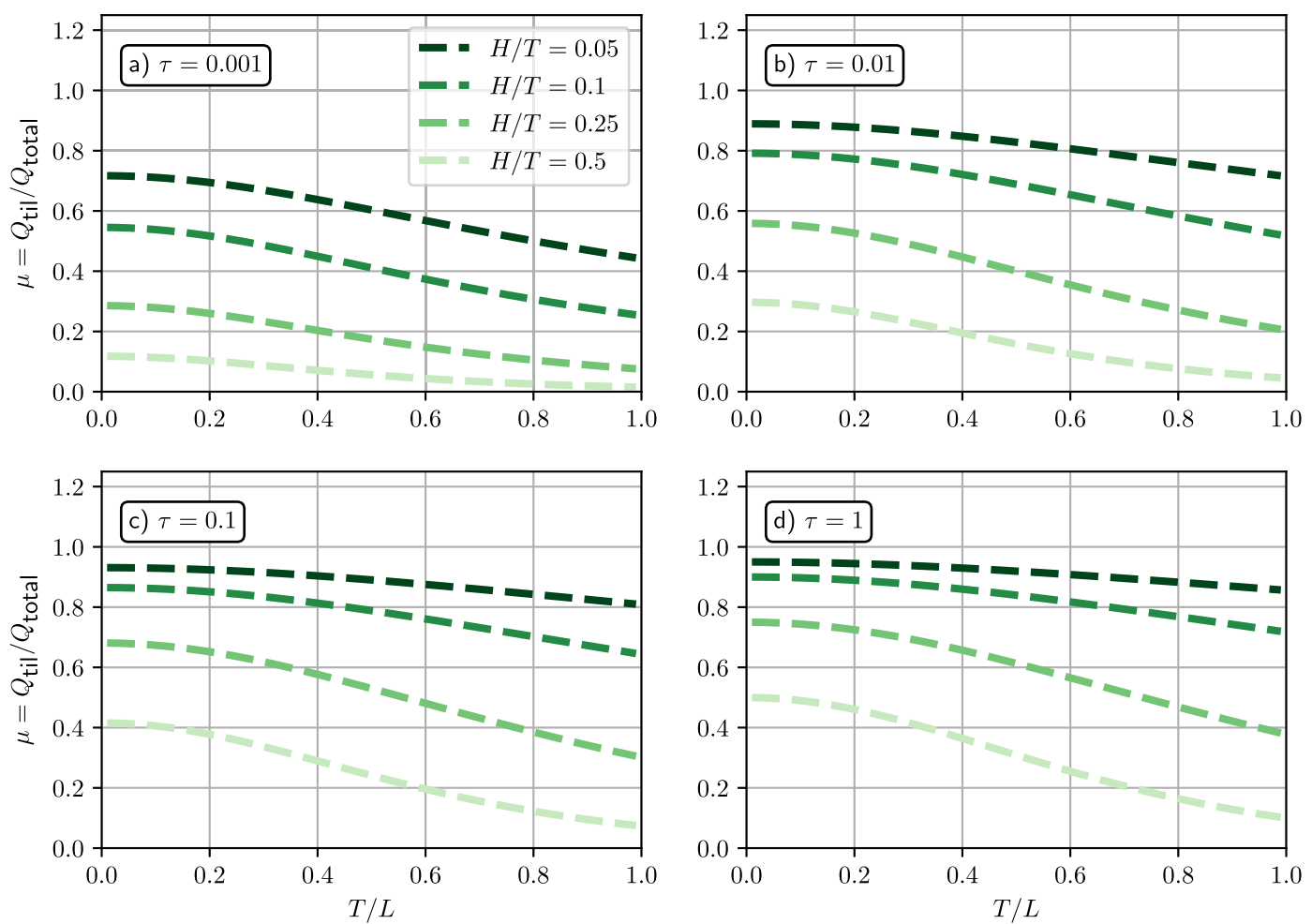

Fig. 8 a-d Dependency of dilution ratio $\mu=Q_{t i l} / Q_{\text {total }}$ on setting parameters of the construction pit for increasing hydraulic conductivity ratios $\tau=K_{\text {til }} /$ $K_{\text {sand }}: T / L$ is the ratio of total depth $T$ to total length $L$ of the domain; $H / T$ denotes the depth of the til $H$ to total depth $T$

for advanced grout layer erosion. Thus, at later stages of erosion, the mass flux entering the aquifer below the construction pit is hardly diluted.

- Mass flux of grout components such as dissolved silicate, sodium, alkaline solution (high $\mathrm{pH}$ ) and dissolved organic matter, increases with ongoing erosion. Thus, environmental impact of the erosion to groundwater quality has the largest effect not directly after construction ceased but a long time after.

- The mass flux out of the construction can be reduced by having a maximally possible distance between the bottom of the vertical walls and the injection layer.

In practice, the analytical solutions allow for a first-order assessment of environmental impact due to leaching of components from a til into the aquifer below construction. Equations (3)-(5) describe the volume flux in case the til has no reduced hydraulic conductivity anymore. Considering that grout material is still present (yet not sealing anymore), it represents a worst-case scenario. The volume flux out of the injected area is highest, whilst no dilution of dissolved erosion products occurs within the construction pit $(\mu=1)$. Equations (6) and (7) describe the volume fluxes at the beginning of the erosion process, representing the lowest volume flux out of the construction pit. Equation (11) can be used to calculate the absolute groundwater flux as function of conductivity ratios for the aquifer and the til being representative for intermediate times. Finally, Eq. (12) provides an estimate of the dilution ratio for a considered conductivity of the til, and consequently allows to calculate the mass flux at every stage of the erosion process.

Results presented in this manuscript are of direct practical use for construction site planning and risk assessment of silicate grout material on urban aquifers. All solutions are functions of the construction pit settings and of the aquifer. They can be used to gain an immediate estimate of fluxes and dilution ratios for any construction site without doing numerical simulations. Since equations allow for a comparison of environmental impact of erosion of the til between various designs, they can be used to engineer an optimum design with regard to risk assessment.

Acknowledgements We thank the associated editor and the reviewers for their helpful comments. The authors further acknowledge CRUX Engineering for their time investment in this research.

Open Access This article is licensed under a Creative Commons Attribution 4.0 International License, which permits use, sharing, adaptation, distribution and reproduction in any medium or format, as long as you give appropriate credit to the original author(s) and the source, provide a link to the Creative Commons licence, and indicate if changes were made. The images or other third party material in this article are included in the article's Creative Commons licence, unless indicated otherwise in a credit line to the material. If material is not included in the article's Creative Commons licence and your intended use is not permitted by statutory regulation or exceeds the permitted use, you will 
need to obtain permission directly from the copyright holder. To view a copy of this licence, visit http://creativecommons.org/licenses/by/4.0/.

\section{References}

Azzam R (2004) Engineering geology for infrastructure planning in Europe: a European perspective. Springer, Heidelberg, Germany, $104 \mathrm{pp}$

Bakker M, Post V, Langevin CD, Hughes JD, White JT, Starn JJ, Fienen MN (2016) Scripting MODFLOW model development using Python and FloPy. Groundwater 54(5):733-739. https://doi.org/10. $1111 /$ gwat. 12413

Bloemendal M, Hartog N (2018) Analysis of the impact of storage conditions on the thermal recovery efficiency of low-temperature ATES systems. Geothermics 71:306-319. https://doi.org/10.1016/j. geothermics.2017.10.009

Curtin D, Peterson ME, Anderson CR (2016) PH-dependence of organic matter solubility: base type effects on dissolved organic C, N, P, and $\mathrm{S}$ in soils with contrasting mineralogy. Geoderma 271:161-172. https://doi.org/10.1016/j.geoderma.2016.02.009

De Zwart AH (2007) Investigation of clogging processes in unconsolidated aquifers near water supply wells. PhD Thesis, Tech. Univ. Delft, The Netherlands

DINOloket (2019) Data and information from the Dutch subsurface. DINOloket. https://www.dinoloket.nl. Accessed 13 December 2019

Eiswirth M, Ohlenbusch R, Schnell K (1997) Use of artificial and natural tracers for the estimation of urban groundwater contamination by chemical grout injections. Tracer Hydrol 97:313-320

Eiswirth M, Hötzl H (2003) Assessing the environmental risk of grouting with soft gels. RMZ-M\&G 50:113-116

Fetter CW, Boving TB, Kreamer DK (2018) Contaminant hydrogeology, 3rd edn. Waveland, Long Grove
Harbaugh AW (2005) MODFLOW-2005, The U.S. Geological Survey modular ground-water model: the ground-water flow process. US Geol Surv Tech Methods 6-A16. https://doi.org/10.3133/tm6a16

Kazemian S, Huat BB, Arun P, Barghchi M (2010) A review of stabilization of soft soils by injection of chemical grouting. Aust J Basic Appl Sci 4(12):5862-5868

Lin WS, Liu CW, Li MH (2016) Influences of specific ions in groundwater on concrete degradation in subsurface engineered barrier system. Springerplus 5(1):745

Littlejohn GS, Concannon M, Wright RH (1997) Engineering properties of silicate-R100 ester chemical grouts. Ground Eng 30(3):36-40

Owusu YA (1982) Physical-chemistry study of sodium silicate as a foundry sand binder. Adv Colloid Interf Sci 18(1-2):57-91. https://doi.org/10.1016/0001-8686(82)85031-8

Powers JP, Corwin AB, Schmall PC, Kaeck WE (2007) Construction dewatering and groundwater control: new methods and applications. Wiley, Chichester, UK. https://doi.org/10.1002/9780470168103

Pujades E, Vàzquez-Suñé E, Carrera J, Jurado A (2014) Dewatering of a deep excavation undertaken in a layered soil. Eng Geol 178:15-27. https://doi.org/10.1016/j.enggeo.2014.06.007

Schnell K (1999) Impact of chemical grout injection on urban groundwater. In: Impacts of urban growth on surface water and groundwater quality: proceedings of an international symposium held during IUGG. IUGG, Birmingham, UK, July 1999, 187 pp

Schnell, K (2001) Hydrogeochemische Prozesse bei Weichgelinjektionen: Stoffbilanzierung, potenzielle Langzeitfolgen und Grundwassergefährdungspotenziale [Hydrogeochemical processes in soft gel injections: material balance, potential long-term consequences and groundwater hazard potential]. PhD Thesis, Karlsruhe Institute of Technology, Karlsruhe, Germany

Spacagna RL, Rasulo A, Modoni G (2017) Geostatistical analysis of settlements induced by groundwater extraction. In: Gervasi $\mathrm{O}$ et al. (eds) Computational science and its applications: ICCSA 2017. Lecture Notes in Computer Science, 10407. Springer, pp 350 364.https://doi.org/10.1007/978-3-319-62401-3_26

Van der Stoel AEC (2001) Grouting for pile foundation improvement. PhD Thesis, Tech. Univ. Delft, The Netherlands 